\title{
RECEPCIÓN CRONOLÓGICA DE LA CRÍTICA LITERARIA SOBRE CUENTOS MALÉVOLOS (1904)
}

\section{CHRONOLOGICAL RECEPTION OF THE LITERARY \\ CRITIQUE ON EVIL TALES (1904)}

\author{
Jesús Miguel Delgado Del Aguila \\ Universidad Nacional Mayor de San Marcos (Lima, Perú)
}

\section{ABSTRACT}

Evil Tales (1904), Clemente Palma's book, reveals a constant topic, based on the terrifying, typical of romanticism. Likewise, that preference of the author is attributed to his affiliation with Edgar Allan Poe, who constructed discourses characterized by the apocalyptic configuration of characters, situations and environments that exhausted the irrational and the paranormal. Since the publication of the compendium of the Peruvian writer, literary exegesis has manifested itself through reviews and prefaces. However, in a second moment, articles and books that investigate the content developed in this work have been made. This procedure of reception of hermeneutics is what prevailed in this study, considering that the main purpose is to identify the persistent guidelines raised by literary criticism, with the aim of establishing thematic aspects that contribute to the understanding of the text and the generation of research. later, distinguished by their delimitation and originality.

Key words: literary analysis, hermeneutics, state of the art, literary criticism, terror 


\section{RESUMEN}

Cuentos malévolos (1904), libro de Clemente Palma, revela un tópico constante, basado en lo terrorífico, propio del romanticismo. Asimismo, aquella preferencia del autor se le atribuye a su filiación con Édgar Allan Poe, quien construyó discursos caracterizados por la configuración apocalíptica de personajes, situaciones y ambientes que desecadenaban lo irracional y lo paranormal. Desde la publicación del compendio del escritor peruano, la exégesis literaria se ha manifestado a través de reseñas y prólogos. Sin embargo, en un segundo momento, se han realizado artículos y libros que investigan el contenido desarrollado en esta obra. Este procedimiento de recepción de la hermenéutica es lo que prevalezco en este estudio, considerando que el propósito principal es identificar los lineamientos persistentes que plantea la crítica literaria, con la finalidad de instaurar vertientes temáticas que contribuyan a la comprensión del texto y la generación de investigaciones posteriores, distinguidas por su delimitación y su originalidad.

Palabras clave: análisis literario, hermenéutica, estado de la cuestión, crítica literaria, terror

Fecha de recepción 5 de septiembre de 2019.

Fecha de aceptación: 22 de noviembre de 2019.

Cómo citar: Delgado Del Aguila, Jesús Miguel (2019): «Recepción cronológica de la crítica literaria sobre Cuentos malévolos (1904)», en Actio Nova: Revista de Teoría de la Literatura y Literatura Comparada, 3: 367-383.

DOI: https://doi.org/10.15366/actionova2019.3.015 


\section{INTRODUCCIÓN}

Preferentemente, esta investigación se enfoca en la corroboración con discursos críticos sobre Cuentos malévolos, la cual se presenta de modo cronológico. Para ello, se busca la repercusión que tuvo el libro en la época (con reseñas y prólogos) y también el desenvolvimiento que se articula en la actualidad (con los análisis de la obra según la hermenéutica literaria). Con el propósito de explicar el tratamiento que hace posible esta indagación, efectúo una estructura particular que se diferencia por tres temas frecuentes: el primero alude sintéticamente al tópico que convencionalmente se desarrolla, además de mencionar a los autores que confrontan con planteamientos relacionados. El segundo abarca los postulados específicos que se manifiestan en sus aportes según el año de publicación. Finalmente, el siguiente se asocia con la metacrítica que elaboro en función de las interpretaciones de los investigadores referidos, con la intención de contribuir en la construcción de la exégesis literaria en torno a esta obra.

El estado de la cuestión que muestro del texto de Clemente Palma cuenta con dos divisiones. La primera se distingue por revelar los estudios primarios que se elaboraron acerca de la recepción inmediata: reseñas y prólogos que fijan las derivaciones del modernismo y la concepción que tiene el autor sobre la realidad (explícita en sus tesis de investigación). La segunda se caracteriza por la inserción de trabajos críticos, como los de Rita Gnutzmann, Peter Elmore, Carlos Eduardo Zavaleta, Gabriela Mora, Elton Honores, entre otros, que tienen en común la libertad de aplicación teórica y conexión con otros contextos y expresiones artísticas.

\section{REPERCUSIÓN DE CUENTOS MALÉVOLOS (1904) EN LA ÉPOCA}

En esta ocasión, se presenta la reseña extraída del diario La Prensa (1904) y los prólogos de Miguel de Unamuno (1904) y Augusto Tamayo Vargas (1974) sobre Cuentos malévolos.

En La Prensa (24 de julio de 1904), existe una reseña anónima hecha en la sección "Lecturas", en función de Clemente Palma y sus tendencias literarias: Cuentos malévolos, opiniones con respecto a su prólogo y temas generales. En esta, se sostiene que el autor erige ideas sugestivas en torno a Filosofía y arte (1897). Asimismo, se destaca el acervo intelectual 
de la raza y el poder analítico, pues él posee flexibilidad para adquirir nuevos conocimientos de las corrientes del pensar moderno. Por otro lado, se prioriza el culto por las energías de la vida en su obra literaria, junto con la concepción misteriosa del amor, entre otros subtemas, como la decadencia, las contradicciones, las referencias indirectas a Nietzsche y los desequilibrios de los personajes. En relación con su tesis doctoral, el cuentista peruano estudia el satanismo y el androginismo: las dos formas que desencadenaron el descuido por lo moderno, además de aproximar su intuición a las psicologías de los personajes y la fantasía (arte de visionario), con percepciones atormentadas, tal como sucede en "Leyenda de Hachisch", en la que se transgrede lo rutinario de la vida y se patentiza lo simbolista, a través de actos insólitos y anómalos. Con ello, se revela las deformidades morales y la psicología de las almas vulgares (considerando el prólogo de Unamuno, en el que se extrapola la noción de "inmoraleja", de la que la malevolencia no está en el espectador, sino en el espectáculo, con una selección de detalles de aspectos sombríos y perversos).

Existen dos prólogos realizados por Miguel de Unamuno (1904) y Augusto Tamayo Vargas (1974). El primero plantea que la malevolencia de la obra de Clemente Palma no es tan sólida, más bien, es humorística. Por ende, vincula al autor con Ricardo Palma. Para él, los relatos no exteriorizan una moraleja, sino una inmoraleja. Igualmente, detecta la negación que tiene hacia Dios, como acaece en "El quinto Evangelio" y "El hijo pródigo", en los que se rechaza la cristiandad tradicional, con contenido moral y teológico: razones para adoptar una postura opuesta a la del cuentista peruano. El segundo fundamenta que Cuentos malévolos es un modelo de texto positivista y modernista, influenciado por temas como los fantasmagóricos o los fantásticos. A la vez, los exponentes son Édgar Allan Poe y Guy de Maupassant. Incluye como ejemplos "La granja blanca", "Los ojos de Lina", "Leyenda de Haschischs" y "Cuento de marionetas".

\section{ESTADO DE LA CUESTIÓN DE CUENTOS MALÉVOLOS (1904) CON RESPECTO A SU REPERCUSIÓN EN LA ACTUALIDAD}

Los estudios realizados sobre la obra de Clemente Palma se han basado en cuatro criterios que servirán para delimitar la investigación del artículo: el primero aborda la temática de los influjos de las tradiciones que ha tenido el escritor peruano para la elaboración de su libro, como los del modernismo, el decadentismo, lo fantástico, lo gótico, el romanticismo y 
la ciencia ficción; el segundo, la ideología que manifiesta el autor en su texto, junto con su participación en lo social; el tercero, los análisis literarios que se han construido en torno a Cuentos malévolos; finalmente, los trabajos que predominan en función de la alusión de lo terrorífico. Después de explicar el rol hecho por los críticos literarios que retoman al cuentista, argumentaré sus propuestas de forma cronológica, con la intención de configurar una metacrítica de las mismas y distinguir las nociones explícitas que se establecerán.

Rita Gnutzmann en Novela y cuento del siglo XX en el Perú (1981) articula la idea de que existe una influencia localizada en Clemente Palma con respecto a nacionalidades y tipos de escritores. Aquello se nota en el primer punto: los narradores rusos tienen una intervención importante en su narración, tal como se aprecia en la estructura del cuento "Los canastos"; la filiación de los franceses Guy de Maupassant y Gérard de Nerva; y la del norteamericano Édgar Allan Poe. Ante estos postulados referenciales, se añade al trabajo de Gnutzmann, el influjo del narrador, según las corrientes o expresiones literarias pertenecientes. Verbigracia, los cuentos de índole modernista latinoamericano formulan una vertiente esotérica y parapsicológica en las narraciones de ese contexto. Para ello, la exégeta compara esa intromisión con los cuestionamientos reconocidos en los relatos de los argentinos Leopoldo Lugones y Horacio Quiroga, junto con los del mexicano Amado Nervo.

Peter Elmore en Clemente Palma: Cuentos malévolos y la cuestión cosmopolita (1984) indica que el escritor peruano erige una narrativa breve y fantástica en su país, además de que para él fue insular. Para validar su argumento, corrobora este libro desde la narratología estructural: se vale de los términos de autor implícito y lector abstracto, junto con una interpretación temática relacionada con la política a su discurso, con el propósito de desligar lo literario de lo social.

Nancy M. Kason en Breaking traditions. The fiction of Clemente Palma (1988) se encarga de dos conclusiones que he asumido como influencias intertextual e intratextual. En una primera oportunidad, la crítica arguye que el autor se basa en elementos estructurales de la ciencia ficción y el modernismo, motivo por el cual no prevalece un interés por representar lo nacional. Por eso, también, se asociará con lo científico y se distanciará de la fe religiosa. Para el caso de la filiación intratextual, la investigadora determina que los cuentos de Palma se derivan de su propio texto Filosofía y arte (1897). Luego de haber procedido con el hallazgo de las vertientes que especifican su propuesta literaria, continúa con el análisis de los mismos, retomando los treinta y dos relatos, que son clasificados según cada inferencia independiente 
entre modernistas, heréticos, decadentes y fantásticos. Posteriormente, repetirá ese procedimiento con sus dos novelas Historias malignas (1925) y XYZ (1935).

Pedro Pablo Viñuales Guillén en su artículo "Clemente Palma, la malicia del Contador" (1991) señala que el autor desarrolla una estética modernista, especialmente, una decadente, la cual es comprobada por la documentación del escritor y el estudio de sus textos, en los que detecta el futurismo en "Nigromante" y "La última rubia"; el doble en "La granja blanca”, etc. Igualmente, el exégeta vincula la idea del satanismo en su obra con los proyectos literarios de Charles Baudelaire, con la intención de reinvertir la moral religiosa, de la que el Demonio es el alma del mundo.

Carlos Eduardo Zavaleta en "Los cuentos de Clemente Palma" (1997) construye tres tópicos principalmente: el estilo, los temas y lo biográfico. El primero consiste en el análisis de las técnicas empleadas en su obra literaria. Por un lado, se fijan los relatos como inferencias erigidas dentro de una extensión media. Por otro, no se configura ni se identifica el narrador en $1 .^{a}$ persona, es decir, no existen demasiados rasgos de subjetividad en los personajes. En su reemplazo, se tomará el narrador en $3 .^{a}$ persona, que se caracteriza por exteriorizar en la narración un trabajo descriptivo y enfocado en la continuidad y fluidez del relato. La segunda concepción que confronta es en función con las temáticas reconocidas en los relatos de Palma. Por ende, cuestiona primordialmente los siguientes contenidos: la lucha del bien contra el mal, la drogadicción, la moral y la religión, los cuales son evidenciados en las investigaciones que hace Zavaleta de los cuentos "Los canastos", "Una historia vulgar", "Los ojos de Lina", "La granja blanca" y "La leyenda del hachisch". Para interés de esta indagación, el exégeta aprecia propiedades terroríficas o mortales que buscan o aspiran la felicidad: tópico que corrobora a partir de "En el carretón", que luego será trasladado y asociado con su libro. Finalmente, menciona al escritor peruano en relación con su biografía, como el contacto que tuvo con Miguel de Unamuno o la trascendencia literaria que le dejó Ricardo Palma.

Gabriela Mora en Clemente Palma. El modernismo en su versión decadente y gótica (2000) y sus artículos "Los Cuentos malévolos de Clemente Palma: integración más allá del bien y del mal" (2006) y "Decadentismo y vampirismo en el modernismo hispanoamericano: un cuento de Clemente Palma" (2007) localiza la sátira al racismo, como se observa en "La última rubia". Con respecto al entorno de la época que se distingue por la ruptura de la moral, la investigadora vincula los temas establecidos de la muerte y la desaparición de Dios en el autor, junto con la filiación ideológica de la filosofía de Nietzsche, tal como lo determina en 
su tesis de bachiller, titulada El porvenir de las razas en el Perú (1897), en la que considera el contexto histórico que ayudó a formar al escritor, con la creencia de que la raza que tenga más vínculos con la española es la superior. En consecuencia, posiciona al indígena en un rango ínfimo. Asimismo, esta idea se reconoce en su tesis doctoral Filosofía y arte (1897), en la que plantea el problema de la cultura en la actualidad peruana, debido al modernismo, junto con la disolución del individualismo y la pérdida de la fe (la religión) (Mora 2000: 34). Por otra parte, logra sintetizar cómo se ha desarrollado la noción de la sexualidad en los personajes femeninos de "Leyenda de Hachisch" y "Los ojos de Lina". También, es importante adoptar que la exégeta configura al cuentista como escritor no canónico (al no ser valorado por la hermenéutica literaria a inicios del siglo XX), decadente (a causa de la inspiración en personajes y autores satánicos, junto con la temática de las drogas) y modernista, que se fundamenta por argumentos contradictorios y ambiguos. No obstante, estas particularidades son más visibles en la posmodernidad (relativismo, fragmentarismo, ruptura de metarrelatos). Por lo tanto, su análisis empieza de forma errónea. Además, Palma rechaza el realismo, el naturalismo y el romanticismo: "La representación positiva de Lucifer —encarnación del Mal- hecha por románticos y decadentes, fue una de las armas de los modernos para atacar los valores burgueses más convencionales" (Mora, 2000: 63). Otra concepción que añade la crítica sobre la base del escritor peruano es el mal insertado en la figura femenina, tal como se aprecia en "Tengo una gata blanca", "Idealismos" y "Los ojos de Lina"; igualmente, lo gótico en "La granja blanca" o "Vampiras"; el exterminio de los cuerpos como una prueba de amor. Un acontecimiento que aumenta el horror en "El príncipe alacrán” es cuando los alacranes aparecen ante el personaje (Mora, 2000: 115-116). Frente a esta propuesta, existe un anacronismo en torno a esa situación: el horror ya no se percibe como antes. De manera semejante, se refiere a "Parábola", "El quinto Evangelio" y "El hijo pródigo" para justificar la manifestación de la figura del mal, personificada a través del propio Diablo. Aquello es influencia de Édgar Allan Poe, incluso de Baudelaire. A ello, se postula el esoterismo (magia, diabolismo y espiritismo).

Clemente Palma en Cuentos malévolos invierte la estética tradicional, en la que lo bello se asocia con lo bueno y lo sagrado (Dios). Este dualismo del bien con el mal se detecta, según Mora (2000: 63), no solo en todas las religiones, sino en las culturas. El escritor peruano muestra un interés por el tema del doble y el conflicto de la identidad desorbitada, porque esta se halla amenazada. Gabriela Mora (2006) distingue sus cuentos según los tópicos. Por ejemplo, infiere que "Las mariposas" y "Cuento de marionetas" tratan de relatos 
infantiles, no de terror, con la evidencia de narradores que se oponen entre sí: en algunos casos, serán confesionales ( $1 .{ }^{a}$ persona) y, en otros, omniscientes $\left(3 .{ }^{a}\right.$ persona), como "El quinto evangelio", "Ensueños mitológicos", "El último fauno" y "Cuento de marionetas". Gabriela Mora (2000) asume que prevalece una preferencia por la ciencia ficción por parte del autor, como se percata en "La última rubia" y "El día trágico". Igualmente, Mora (2006) diferencia sus cuentos según las temáticas. Al respecto, formula que "Las mariposas" y "Cuento de marionetas" abordan relatos infantiles, no terroríficos.

Ricardo Sumalavia en "Clemente Palma y el modernismo peruano: la búsqueda del ideal” (2006) y La prose moderniste péruvienne et la vision de la modernité chez. Manuel González Prada, Clemente Palma et Ventura García Calderón (2014) posiciona al autor como un exponente del modernismo hispanoamericano, debido a que se observa la representación de la filosofía y los elementos malignos. Intuye el influjo de Édgar Allan Poe, explícitamente en La caída de la casa Usher (1839), en la que se percatan las ideas del doble y el incesto; también, la filiación con el romanticismo. En consecuencia, existe una visión reaccionaria (personaje mira hacia el pasado). Según Sumalavia (2014), se aprecia una crisis de lo tradicional con el escritor peruano, ya que se prioriza la exhibición de rasgos románticos, sin necesidad de que se abarque este tópico en su totalidad. Asimismo, localiza particularidades de la ciencia ficción en "La última rubia" e influencia del modernismo latinoamericano y el decadentismo europeo en este libro.

Moisés Sánchez Franco en La representación del sujeto aristócrata y del sujeto juvenil drogado en Historietas malignas de Clemente Palma (2007) recurre al concepto de modernismo para explicar la obra del escritor peruano. Además, realiza un estado de la cuestión del autor, enfocándose en Historietas malignas y la noción de drogadicción.

Gonzalo Cea Monsalves en “Tentación, agonía y muerte en 'Idealismos' de Clemente Palma” (2009) y "La intertextualidad en dos cuentos heréticos de Clemente Palma” (2015) corrobora únicamente "Idealismos", del cual hace un resumen para luego cuestionarlo desde las acepciones del amor y la sexología. Para ello, se basa el argumento de Michael Foucault para defender la tesis de que el acto sexual es peligroso y atenta contra la libertad, propuesta que se desarrolla efectivamente en el cuento. El estudio de Gonzalo Cea Monsalves (2009) defiende la postura equivocada de uno de los personajes, puesto que se conoce que el protagonista mantiene una idea errada de la existencia y el amor, pero no es vinculada y sustentada con el significado del terror. Por lo tanto, se delimita bien qué se entiende por el terror, sin necesidad de designar al autor una corriente específica, sino 
distanciarlo para observar los elementos que configuran espacios sobrenaturales y malévolos. Esto se alcanza con una documentación y una subdivisión del concepto de terror en la literatura. Más adelante, Cea Monsalves (2015) se basa en lo religioso (con la figura de Cristo), desde lo intertextual (Julia Kristeva), para entender la estética del cuentista (la cual se caracteriza por ser decadentista del modernismo y adquirir la tradición literaria mitológica y cristiana), con la finalidad de plantear un procedimiento herético y de desacralización al reescribir el discurso religioso. Aquello es demostrado en "El Quinto Evangelio", al erigir la última tentación que tuvo Cristo, junto con la mención de la obra de Miguel de Cervantes y la inserción de Satanás como visionario en el calvario.

María Julia Olijnyk en "Visiones de amor y muerte en dos Cuentos malévolos de Clemente Palma" (2014) confronta "El día trágico" e "Idealismos". Para ello, retoma la idea de que el autor es modernista y decadentista, y que su producción se constituye en torno a aquellas nociones, porque manifiesta temas asociados con la muerte y el amor distorsionado.

Elton Honores en La civilización del horror (2013) señala que Palma recurre a rasgos comunes de la narrativa nacional en dicho período, además de ubicarlo en el primer horizonte de la literatura peruana de terror (fines del siglo XIX hasta 1940), ya que alude al género fantástico o relato de terror, junto con tópicos como el mal, el sexo, la mujer de manera negativa y los orígenes bíblicos. Para comprobar esta formulación, el crítico corrobora "La granja blanca" de Cuentos malévolos y su novela Mors ex vita (1923) para detectar propiedades religiosas a través de lo fantástico y lo perverso.

Wilmer Ventura en El pensamiento estético de Clemente Palma en dos ensayos: Excursión literaria y Filosofía y arte (2014) se sirve del análisis que hizo Gabriela Mora. Sin embargo, lo innovador de su fundamentación es la de indagar la obra ensayística del autor, en especial, su tesis doctoral, para después interpretar sus cuentos, como lo hace con "Las vampiras", con el objetivo de demostrar cómo los personajes portan valores o posiciones filosófico-éticas. Por ende, resulta simbólico en relación con su ideología. Por otro lado, arguye que el autor se distancia de los temas nacionales. Esto provoca su tránsito al modernismo, lo exótico y lo fantástico.

Encarnación López Gonzálvez en su artículo "De la tradición gótica en la literatura hispanoamericana: 'La Granja Blanca', de Clemente Palma” (2014) se refiere al autor como gótico de corte anglosajón, por la presencia de elementos necrofílicos, incesto y ocultismo, que se distinguen, a la vez, por ser transgresores, incontables, productores de temor y que se desplazan por espacios oscuros. Por otra parte, identifica el influjo de Édgar Allan Poe (por 
"El retrato oval", "Ligeia" y "Morella"), debido a la configuración de sus espacios, sus situaciones y su construcción de la mujer. Por consiguiente, logra esas conexiones intertextuales literarias.

Mateo Díaz Choza en La secularización en los ensayos y la narrativa de Clemente Palma (2015) diferencia y explica las diversas clasificaciones que ha tenido el cuentista peruano, como las de posicionarse dentro del modernismo, el decadentismo, el ateísmo y el anticlericalismo, desde la hermenéutica social del libro y la teoría de los campos figurativos de la retórica general textual. También, detalla el proceso de secularización que posee el autor, del cual se entiende la muerte de Dios, según Nietzsche, la sacralización del mundo, la exposición literaria del Diablo, entre otras inferencias que aluden a la obra completa del escritor. Esta secularización, según el investigador, no ha sido abordada por la exégesis de una forma tan concisa. Por otro lado, asume que sus textos revelan una preferencia por la religión, para cuestionarla (propio del ateísmo): no adopta una postura de creyente. Para vincular ese contenido, analiza los cuentos "Parábolas", "El día trágico", "El quinto evangelio", "El hijo pródigo", "El último fauno" y "Ensueños mitológicos”, en los que se incorpora la lógica secular: se pregunta sobre la existencia de Dios y el cristianismo.

Considerando los cuatro tópicos que se extraen de este estudio cronológico realizado de Clemente Palma, en función de Cuentos malévolos, se halla un desligamiento de las tradiciones para captar el concepto variable de terror, que es percibido por los lectores actuales de esta obra, aunque intuir las filiaciones es requisito para entender el pensamiento y los intereses del autor (el romanticismo de Édgar Allan Poe). Sin embargo, no resulta prioritario defender ni clasificar los cuentos del escritor por tradiciones. En segundo lugar, su ideología es fundamental para reconocer su crítica a propósito de la sociedad, puesto que se distancia de su contexto para optar modelos extranjeros, a pesar de que no se separa completamente, a causa de que abarca la moralidad peruana a través de contenidos que atacan su susceptibilidad (la religión, la moral, la convivencia). En tercer lugar, los análisis efectuados por lo literario mejoran su calidad y permiten la difusión de la diversidad de aplicaciones obtenidas de los cuentos y la obra en general de Clemente Palma. Finalmente, con respecto a las aproximaciones hechas al autor en torno a los elementos de terror y maldad pertenecientes (específicamente, en su libro), se deduce la predominancia de una posibilidad de embellecer el texto con conductas anormales (todas relacionadas con lo terrorífico). No obstante, se intuye de todas maneras una carencia o una ausencia de correspondencia en la actualidad. Esto es debido a que esta emoción ya no repercute de forma similar como sucedía 
hace un siglo atrás, porque las personas han ido perdiendo sensibilidad en ese aspecto receptivo (premisa que no rescató Gabriela Mora, ya que ella asume que sí se localiza el terror en muchos de los cuentos). Hasta el momento, no se ha delimitado correctamente qué se entiende por terror (una visión diacrónica y contemporánea del mismo). Por el contrario, sí se precisan temas en los estudios de la crítica literaria que se manifiestan, pero no son vinculados correctamente con el principal: prevalece una noción incompleta del terror. Ante esta situación, en este artículo, faltaría investigar e indagar el terror desde lo teórico para complementarlo con distintos postulados.

En suma, existen dos criterios que ha consolidado la exégesis: el estudio de las técnicas y la conceptualización que tiene el escritor peruano del modernismo y el decadentismo. En primer lugar, Carlos Eduardo Zavaleta (1997) se enfoca en la investigación de las técnicas narrativas, que a mi criterio no es una característica primordial de Clemente Palma, sino que desarrolla y corrobora el contenido propuesto y tratado por Édgar Allan Poe. Por ende, es provechoso partir de la bibliografía primaria, es decir, los escritos por el cuentista (quien hizo dos tesis en torno a las ciencias ocultas y el racismo: temas polémicos y equidistantes para la moralidad de la época). En segundo lugar, ante las fundamentaciones de análisis mencionados, resta hacer una conceptualización del terror, por lo observado en los estudios de Pedro Pablo Viñuales Guillén (1991), Gabriela Mora (2006) y Ricardo Sumalavia (2006), en los que se retoman la noción y la contextualización artísticas, pues estas catalogan al autor como difusor del modernismo y el decadentismo, aunque no se establece la idea del terror, a pesar de evidenciarse tópicos que no se relacionan con el principal.

\section{CONCLUSIONES}

La crítica literaria que se erige en función de Cuentos malévolos (1904) es provechosa a causa de que logra representar particularidades que ubican al autor en una determinada ideología con patrones que caracterizan su obra en general. Los reiterados aportes permitieron que se construya un panorama relacionado con las investigaciones expuestas por la exégesis. A la vez, apertura la posibilidad de que se ejerzan indagaciones y cuestionamientos en torno a lo tratado en este artículo, como también postular concepciones, interpretaciones o estudios sobre la base de lo que resta por analizar de este libro. 
Con respecto a lo corroborado, exhibiré sintéticamente los resultados obtenidos de la hermenéutica del compendio publicado por Clemente Palma. En primer lugar, se deriva de la reseña y los prólogos de este texto la transferencia ideológica que persiste en la obra del escritor peruano por la incorporación de rasgos de Filosofía y arte (1897) y discursos similares, en los que se muestra el pensamiento moderno, con filiación anárquica y despectiva de la sociedad. Equivalentemente, los temas vinculados con el satanismo están exteriorizados como interés permanente. Por ese motivo, es obvio inferir que la cosmovisión predominante es la de la inmoraleja y la perversidad. En segundo lugar, distintos críticos literarios hicieron referencia al cuentista peruano para asociarlo con otros autores, como Édgar Allan Poe, Guy de Maupassant, Gérard de Nerva, Leopoldo Lugones, Horacio Quiroga, Amado Nervo. Asimismo, es oportuna la ocasión para aplicar conceptos teóricos, como el de identificar las técnicas narrativas, el estilo o los narradores, al igual que el contenido, distinguido por el romanticismo, el modernismo, el decadentismo, el ateísmo y el terror. 
BIBLIOGRAFÍA

Cea Monsalves, Gonzalo (2009): «Tentación, agonía y muerte en "Idealismos” de Clemente Palma». Atenea, I semestre, (499): 149-156.

Cea Monsalves, Gonzalo (2015): «La intertextualidad en dos cuentos heréticos de Clemente Palma», en Letras, 86 (123): 69-82.

Díaz Choza, Mateo (2015): La secularización en los ensayos y la narrativa de Clemente Palma, Tesis de Licenciatura, Universidad Nacional Mayor de San Marcos de Lima, Perú.

El Libre Pensamiento (6 de febrero de 1904): «Estúpido y abominable negocio de los padres salesianos en el Callao. Deificación y fraudes clericales», en ElLibre Pensamiento, VIII (402).

El Libre Pensamiento (13 de febrero de 1904): «Barbaries del clericalismo» e «Inmoralidad de las procesiones de iglesia. Abolición y prohibición de ellas en la República de Bolivia», en El Libre Pensamiento, VIII (403).

El Libre Pensamiento (12 de marzo de 1904): «El fanatismo religioso», en El Libre Pensamiento, VIII (407).

El Libre Pensamiento (2 de abril de 1904): «La Iglesia Romana no es cristiana ni católica, es pagana», en El Libre Pensamiento, VIII (410).

El Libre Pensamiento (18 de junio de 1904): «Porvenir de las religiones», en El Libre Pensamiento, VIII, (421).

El Libre Pensamiento (15 de octubre de 1904a): «La mujer en el Código y en la Sociedad I» y «Una nueva religión», en El Libre Pensamiento, IX (438).

El Libre Pensamiento (29 de octubre de 1904): «La mujer en el Código y en la Sociedad II», en El Libre Pensamiento, IX (439).

El Libre Pensamiento (5 de noviembre de 1904): «La mujer en el Código y en la Sociedad III», en El Libre Pensamiento, IX (441).

El Libre Pensamiento (31 de diciembre de 1904): «Un templo masónico en Lima», en El Libre Pensamiento, IX (489).

Elmore, Peter (1984): Clemente Palma: Cuentos malévolos y la cuestión cosmopolita, Tesis de Bachiller, Pontificia Universidad Católica del Perú de Lima, Perú.

Gnutzmann, Rita (1981): Novela y cuento del siglo XX en el Perú, núm. 21, prólogo de José Morales Saravia, Alicante, Universidad de Alicante, Cuadernos de América sin nombre. 
Honores, Elton (2013): La civilización del horror, Lima, El Lamparero Alucinado.

Kason, Nancy (1988): Breaking traditions. The fiction of Clemente Palma, Lewisburg, Bucknell University Press.

López Gonzálvez, Encarnación (2014): «De la tradición gótica en la literatura hispanoamericana: "La Granja Blanca", de Clemente Palma», en Brumal. Revista de Investigación sobre lo Fantástico, II (2): 177-186.

Los Parias (1 de mayo de 1905): «Los sacerdotes», en Los Parias, II (13).

Los Parias (junio de 1905): «La voluntad de Dios», en Los Parias, II (14).

Los Parias (octubre de 1905): «Las víctimas de los accidentes» y «Horrores en el Acre», en Los Parias, II (18).

Miró Quesada, José (dir.) (10 de enero de 1900): «Fiestas religiosas» e «Inserciones. La conferencia del doctor patrón», en El Comercio, (23).

Mora, Gabriela (2000): Clemente Palma. El modernismo en su versión decadente y gótica, Lima, Instituto de Estudios Peruanos.

Mora, Gabriela (2006): «Los Cuentos malévolos de Clemente Palma: integración más allá del bien y del mal» en Brescia, P. y Romano, E. (coords.), El ojo en el caleidoscopio, México, Textos de Difusión Cultural, Universidad Autónoma de México.

Mora, Gabriela (2007): «Decadentismo y vampirismo en el modernismo hispanoamericano: un cuento de Clemente Palma», en Revista de Crítica Literaria Latinoamericana, año XXII (46): 191-198.

Morales de la Torre, Pedro (15 de mayo de 1909): «La muerte», en V ariedades, V (63): 259.

Olijnyk, María Julia (2014): «Visiones de amor y muerte en dos Cuentos malévolos de Clemente Palma», en III Congreso Internacional Cuestiones Críticas, Rosario, Centro de Estudios de Literatura Argentina, Centro de Estudios de Literatura y Crítica Literaria: https://bit.ly/34fAIEg (último acceso 5/9/2019).

Palma, Clemente (1904): Cuentos malévolos, Barcelona, Impresión Salvat.

Palma, Clemente (dir.) (11 de abril de 1908): «Almas que emigran», «Otras almas» y «Los habitantes de Marte», en Variedades, IV (6).

Palma, Clemente (dir.) (18 de abril de 1908): «La erección de la Cruz», en V ariedades, IV (7).

Palma, Clemente (25 de abril de 1908): «Carta literaria», en V ariedades, IV (8).

Palma, Clemente (dir.) (1 de agosto de 1908): «Cuentos malévolos por Clemente Palma (ed. de Barcelona) con prólogo de Miguel de Unamuno. - El tomo empastado se vende al precio de dos soles en las librerías de Rosa y de Gil», en V ariedades, IV (22): 736. 
Palma, Clemente (dir.) (13 de febrero de 1909): «En el mundo de las hadas y los duendes», en Variedades, IV (50): 1623-1624.

Palma, Clemente (dir.) (26 de junio de 1909): «Los últimos suicidios», en Variedades, V (69): 393.

Palma, Clemente (dir.) (25 de septiembre de 1909): «El crimen Roccatagliata», en Variedades, $\mathrm{V}(82): 711$.

Palma, Clemente (dir.) (15 de octubre de 1909): «El fusilamiento de Ferrer», en Variedades, V (85): 775-776.

Palma, Clemente (dir.) (30 de octubre de 1909): «Almas mudas», en V ariedades, V (87): 838.

Palma, Clemente (dir.) (2 de abril de 1910): «La semana santa», «La fiesta de los niños pobres en Miraflores»y «Los días santos en Chorrillos», en Variedades, VI (109): 420-424.

Palma, Clemente (dir.) (10 de septiembre de 1910): «Espiritismo», en Variedades, VI (132): 1137.

Palma, Clemente (1912): Cuentos malévolos, París, Lib. Paul Ollendorff.

Palma, Clemente (1959): Cuentos malévolos, colección dirigida por José Bonilla Amado, Lima, Editorial Nuevos Rumbos.

Palma, Clemente (1974): Cuentos malévolos, Lima, Ediciones Peisa.

Palma, Clemente (2006): Obras esenciales. Prólogo y cronología de Ricardo Sumalavia, Lima, Pontificia Universidad Católica del Perú.

Sánchez Franco, Moisés (2007): La representación del sujeto aristócrata y del sujeto juvenil drogado en Historietas malignas de Clemente Palma, Tesis de Licenciatura, Universidad Nacional Mayor de San Marcos de Lima, Perú.

Sumalavia, Ricardo (2006): «Clemente Palma y el modernismo peruano: la búsqueda del ideal» en Palma, Clemente, Obras esenciales. Prólogo y cronología de Ricardo Sumalavia. Tomo I, Lima, Pontificia Universidad Católica del Perú: 9-39.

Sumalavia, Ricardo (2014): La prose moderniste péruvienne et la vision de la modernité chez. Manuel Gonqález Prada, Clemente Palma et Ventura García Calderón, Tesis Doctoral, Université Michel de Montaigne de Bordeaux, Francia.

Ulloa Cisneros, Alberto (dir.) (4 de febrero de 1905): «El cadáver de Dulanto abandonado. No existe Morgue en el Callao», en La Prensa, (715), ed. de la tarde.

Ulloa Cisneros, Alberto (dir.) (18 de febrero de 1905): «Crimen por celos. Drama pasional. Dos puñaladas. Captura del criminal», en La Prensa, (739), ed. de la mañana. 
Ulloa Cisneros, Alberto (dir.) (25 de febrero de 1905): «Asalto y robo en la Magdalena Vieja», en La Prensa, (753), ed. de la mañana.

Ulloa Cisneros, Alberto (dir.) (1 de marzo de 1905): «Un guardia criminal en la comisaría del 6. ${ }^{\circ}$. Captura», en La Prensa, (760), ed. de la mañana.

Ulloa Cisneros, Alberto (dir.) (3 de marzo de 1905): «Caso de locura. En un calabozo de la Intendencia. Comiéndose a un perro», en La Prensa, (765), ed. de la tarde.

Ulloa Cisneros, Alberto (dir.) (22 de mayo de 1905): «Suicidio en la Maisón de Santé. Un enfermo que se ahorca», en La Prensa, (908), ed. de la mañana.

Ventura, Wilmer (2014): Elpensamiento estético de Clemente Palma en dos ensayos: Excursión literaria y Filosofía y arte, Tesis de Licenciatura, Universidad Nacional Mayor de San Marcos de Lima, Perú.

Viñuales Guillen, P. (1991): «Clemente Palma, la malicia del contador», en Anales de Literatura Hispanoamericana, (20): 103-118.

Zavaleta, Carlos Eduardo (1997): «Los cuentos de Clemente Palma» en El gozo de las letras, Lima, Pontificia Universidad Católica del Perú, Fondo Editorial: 35-49. 
SOBRE EL AUTOR

\section{Jesús Miguel Delgado Del Aguila}

Candidato a doctor en Literatura Peruana y Latinoamericana por la Universidad Nacional Mayor de San Marcos (Lima, Perú), además de ser investigador literario, con intereses en la narrativa y los ensayos de autores latinoamericanos, como Mario Vargas Llosa, Gabriel García Márquez y Octavio Paz. Se ha desempeñado como docente de Educación Superior y universidades nacionales; asimismo, ha trabajado como corrector de textos en editoriales. Cuenta con artículos publicados en revistas nacionales e internacionales, indexadas en Web of Science, SciELO y Scopus. De igual manera, ha sido ponente en congresos literarios de su localidad y otros países.

Contact information: Correo electrónico: tarmangani2088@outlook.com 